Open Access

\title{
Should thorough Debridement be used in Fibular Allograft with impaction bone grafting to treat Femoral Head Necrosis: a biomechanical evaluation
}

Guangquan Zhou ${ }^{1,2}$, Ying Zhang ${ }^{2,4}$, Linghong Zeng ${ }^{5}$, Wei He ${ }^{1 * \dagger}$, Zhihui Pang ${ }^{2^{*+}}$, Xiumin Chen ${ }^{3^{*+}}$, Yujing Xu' Liao Shaoyi Stephen ${ }^{6}$ and LeiLei Chen ${ }^{1}$

\begin{abstract}
Background: Fibular allograft with impaction bone grafting (FAIBG) is an effective hip-preservation method for avoiding total hip arthroplasty in the early stage of femoral head necrosis. However, whether thorough debridement should be used with FAIBG is controversial. This study compared the mechanical performance between FAIBG with and without thorough debridement, which provides a biomechanical basis for selecting the proper treatment in clinical settings.
\end{abstract}

Methods: Eighteen computational models were constructed and used to simulate two subtypes of femoral head collapse with seven debridement radii. The initial model was validated using the bony density distribution from $X$-ray images and a photograph of the cadaver bone cross-section. The stress of the anterolateral column and the debridement efficiency were computed and analyzed.

Results: (1) The peak stress of the anterolateral column in all conditions could return to the physiological level, and in two cases, the decrement/increment of stress was almost less than $0.1 \%$ when the debridement radius increased. (2) The load share ratio (LSR) of the cortical and cancellous bone was markedly decreased in the untreated condition and increases with an increase in the debridement radius. (3) A debridement radius greater than $1 / 2 r$ yields a LSR value larger than that obtained in the normal condition.

Conclusions: The simulation results provide specific biomechanical evidence to support the finding that FAIBG with a debridement region of 3/8 -1/2 appears to be a better choice for resisting femoral head collapse (FHC). Furthermore, FAIBG without thorough debridement, which requires relatively simple surgical devices and reduces artificial damage, appears to be a better method for resisting FHC than FAIBG with thorough debridement.

Keywords: Computational biomechanics, Thorough debridement, Stress transfer path, Load share ratios, Stress shielding

\footnotetext{
* Correspondence:

157527262@qq.com; 1963675306@qq.com; 104652014@qq.com

${ }^{\dagger}$ Equal contributors

'The First Affiliated Hospital, Guangzhou University of Chinese Medicine, Guangzhou, China

${ }^{2}$ Laboratory of National Key Discipline Orthopaedics and Traumatology of

Chinese Medicine, Guangzhou University of Chinese Medicine, Guangzhou,

China

${ }^{3}$ Department of Rheumatology, Guangdong Provincial Hospital of Chinese

Medicine, China and Postdoctoral Mobile Research Station, Guangzhou

university of Chinese Medicine, Guangzhou, China

Full list of author information is available at the end of the article
} 


\section{Background}

The incidence of femoral head necrosis (FHN) is rapidly increasing worldwide because of the widespread use of steroids [1,2] and alcohol [3-6]. FHN is associated with high morbidity and disability. Patients with FHN are often at high risk of femoral head collapse (FHC), arthritis or disarticulation, which finally results in hip replacement (HR). Statistical data show that the medium- and longterm effects of hip-implant are obviously unsatisfactory; thus, young patients with HR will require several surgical treatments [7]. Hence, various head-preserving procedures have been developed to protect the femoral head of patients and avoid HR, particularly in the early stage of FHN.

Fibular allograft with impaction bone grafting (FAIBG) is an effective head-preservation method for avoiding HR in the early stage of FHN. The advantage of this hippreservation method is that it provides both repaired materials and biomechanical structural support during the healing of the necrosis region [8-11]. However, the disadvantage in using the FAIBG procedure lies in the fact that wide debridement may increase the incidence of cartilage injury and the strength of impaction bone grafting is difficult master. Hence, whether thorough debridement should be used with FAIBG is controversial. "With thorough debridement" indicates that the necrotic bone should be completely cleaned, whereas "without thorough debridement" indicates that the necrotic bone should undergo partial debridement. In most cases, the choice is based on the experience and preference of the surgeon without scientific evidence. Simultaneously, relatively few studies have compared the risk of postoperative FHC with and without thorough debridement.

The clinical practice concept requires theoretical proof. This study presents two subject-specific FHN cases without FHC to compare the mechanical performance between FAIBG with and without thorough debridement, which provides a biomechanical basis for selecting the proper treatment in clinical settings.

\section{Methods \\ JIC Classification}

In 2001, the Japanese Investigation Committee (JIC) [12] revised the diagnostic criteria used to clarify the definition of osteonecrosis of the femoral head (ONFH). According to the JIC classification criteria, FHN is classified into subtypes $\mathrm{A}, \mathrm{B}, \mathrm{C} 1$ and $\mathrm{C} 2$ based on the location of the lesion in the weight-bearing area. Type A lesions occupy the medial one-third or less of the weight-bearing portion, type B lesions occupy the medial two-thirds or less of the weight-bearing portion, type $\mathrm{C} 1$ lesions occupy more than the medial two thirds of the weight-bearing portion but do not extend laterally to the acetabular edge, and type C2 lesions occupy more than the medial two-thirds of

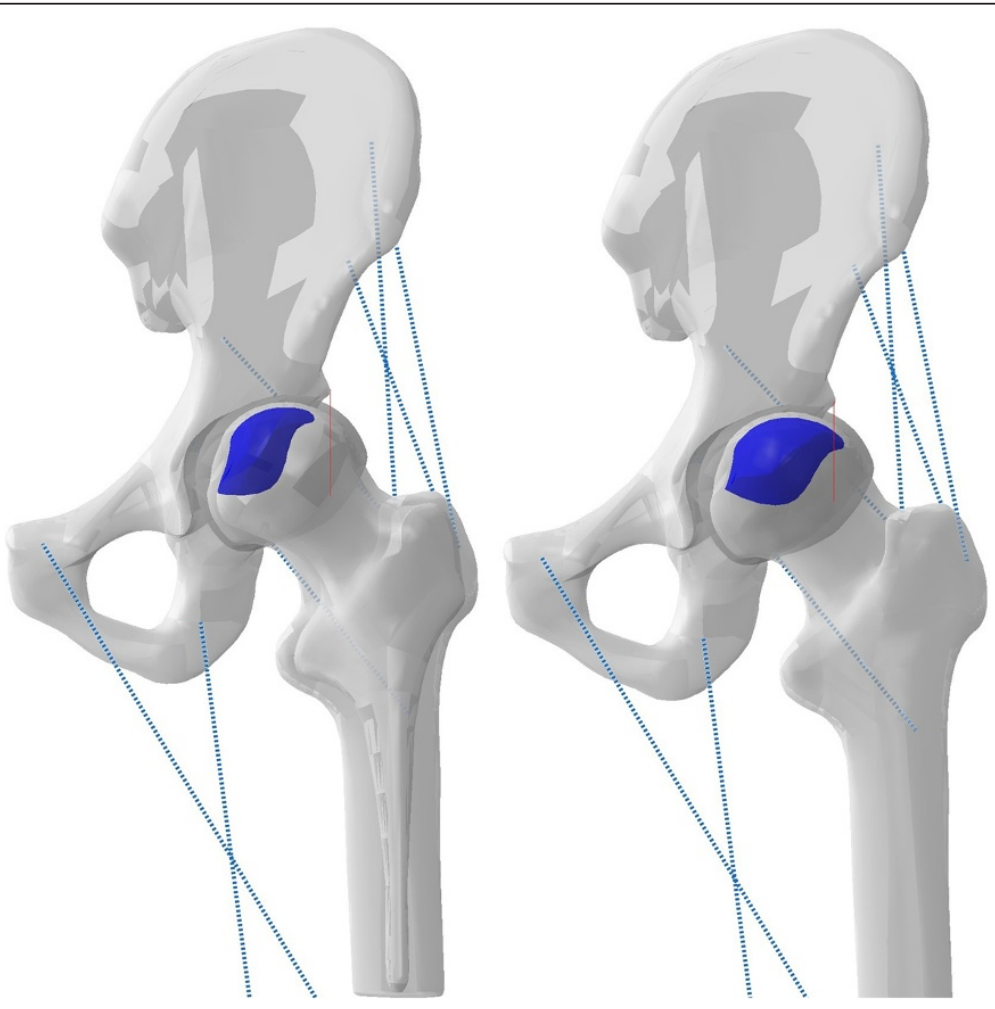

Fig. 1 3D subtype models of FHN 
the weight-bearing portion and extend laterally to the acetabular edge.

Recent studies show that patients who conform to the JIC C criteria are suitable for FAIBG. However, these conclusions are mainly based on clinical observation experience and must be proven both theoretically and in practice. Whether thorough debridement should be used with FAIBG remains controversial. We postulated that the FAIBG procedure with different debridement regions results in different biomechanical performances, which could affect the choice of treatment procedure for FHN. Hence, we reconstructed two subject-specific models (JIC C1 and C2, Fig. 1) to provide a biomechanical basis for exploring the performance of FAIBH with different debridement regions for the treatment of FHN [Ethical approval was granted by the local ethics committee (Constitution of the medical ethics committee: The First Affiliated Hospital, Guangzhou University of Chinese Medicine). Written informed consent for participation in the study was obtained from participants.].

\section{Generation of Intact Finite Element Models}

A JIC C1 FHN-diagnosed patient (P1, last name Fu) with a weight of $70 \mathrm{~kg}$ and a JIC C2 FHN-diagnosed patient (P2, last name Wan) with a weight of $60 \mathrm{~kg}$ were selected for the biomechanical evaluation of the proximal femur (informed consent was obtained from all patients). Computed tomography datasets $(0.5 \mathrm{~mm}$ thickness; Toshiba Aquilion 64, Japan) for each case were used to reconstruct solid models with grey-level processing with the MIMICS 15.1 software based on the "Thresholding", "Edit Masks", and "Calculate 3D" functions. The solid models in the STL format were inputted into the Rapidform preprocessor, and surface-fitting was then performed. Based on the "Mesh" and "Autosurfacing" functions, we found the fit hip to generate the NURBS models. The interface between the ilium and femoral head was used to identify the cartilage geometry. All NURBS models in the igs format were inputted into ABAQUS V6.13 (SIMULIA co., France) to generate nonlinear elastic finite element models. Based on the initial hip geometry, we simulated physiological and pathological models using different materials.

All of the models were then inputted into ABAQUS V6.13 to generate isotropic 10-node tetrahedral elements with a mesh size of $4 \mathrm{~mm}$. The initial models consisted of various elements (146879 in P1; 156471 in P2) and nodes (213970 in P1; 230541 in P2). In these models, the single-legged stance was considered a representative body position, and a ground reaction force equivalent to

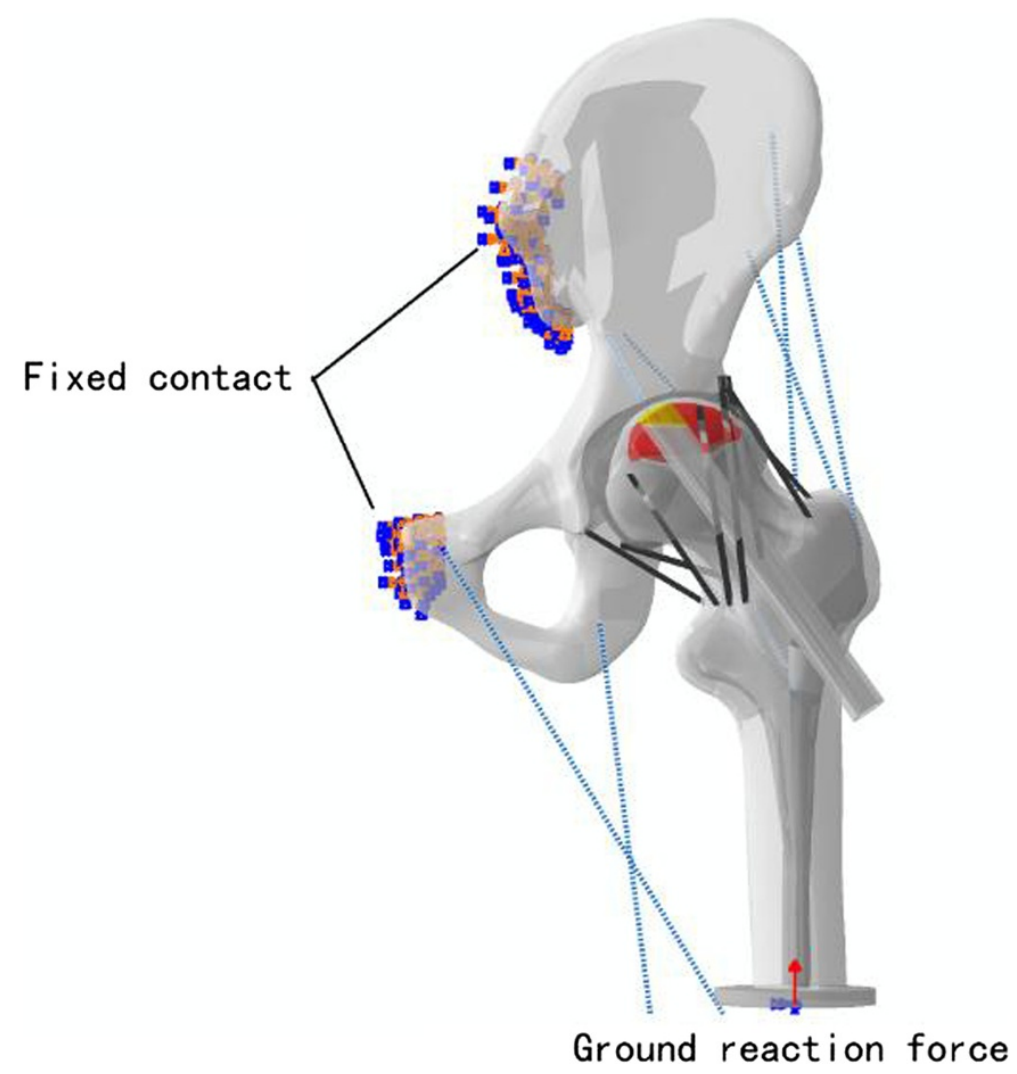

Fig. 2 Load and constraint conditions 
the body weight was performed on a rigid plate, which was tied to the distal part of the femur in Fig. 2. Constraints were applied to the pubic symphysis and sacroiliac joint. All six degrees of freedom were constrained to zero. Seven muscles were modeled as axial connectors, and the muscle forces were set according to the literature [13]: adductor longus $=560 \mathrm{~N}$; adductor magnus $=600 \mathrm{~N}$; gluteal maximus $=550 \mathrm{~N}$; gluteal medius $=700 \mathrm{~N}$; gluteal minimus $=300 \mathrm{~N}$; piriformis $=500 \mathrm{~N}$; tensor fascia latae $=300 \mathrm{~N}$. The models consist of cortical, trabeculae, cartilage and lesion bone. The material properties used in the biomechanical experiment were obtained from the literature [14-16]: Ecortical $=15,100 \mathrm{MPa}$, Etrabeculae $=445 \mathrm{MPa}$, Ecartilage $=10.5 \mathrm{MPa}$, Elesion $=$ $124.6 \mathrm{MPa}$, vcortical $=0.3$, vtrabeculae $=0.22$, vcartilage $=$ 0.45 and vlesion $=0.152$.

The parametric analysis was designed to explore the effects of the extent of debridement of necrotic bone in cases that require surgery. The maximum debridement radius was defined as $\mathrm{r}$, and the debridement extent variants are schematically shown in Fig. 3 . We assumed that the anterolateral cortical stress corresponding to the debridement extent of the necrotic lesion had an increased radius $R(R=1 / 4 \mathrm{r}, 3 / 8 \mathrm{r}, 1 / 2 \mathrm{r}, 5 / 8 \mathrm{r}, 3 / 4 \mathrm{r}, 7 / 8 \mathrm{r}$ and $\mathrm{r})$, where $R=1 / 4 r$ refers to the least debridement and $R=r$ denotes thorough debridement. To simulate the allogeneic fibular implant, the dimensions (length $=80 \mathrm{~mm}$ and radius $=6 \mathrm{~mm}$ ) were obtained from the manufacturer. The axial direction of the fibula was defined by the entry point and lesion centroid. The entry point was located in the trochanteric lateral cortex of the femur. The distance of the cortical bone from the apex of the fibula was $5 \mathrm{~mm}$. The remaining voids were occupied by impaction cancellous bone after the debridement.

\section{Results}

\section{Stress transfer path}

The principal stress transfer characteristics are the most important biomechanical index in the performance evaluation of FHN. In all femoral heads, the principal stress transfer patterns were computed during a gait midstance. Figs. 4a and c show that the principal stress distributions in healthy conditions are from the top of the femoral head to the femoral calcar. As shown in Figs. $4 \mathrm{~b}$ and d, the stress transfer paths are broken off, and the areas that bear the principal stress are less than approximately $50 \%$ of the healthy simulations. The principal stress transfer efficiency markedly decreased.

\section{Stress of the anterolateral column}

FAIBG presents a considerably small risk of structural collapse compared with the untreated situation. Fig. 5 shows the relationship between anterolateral stress and the debridement region. The maximum stress values

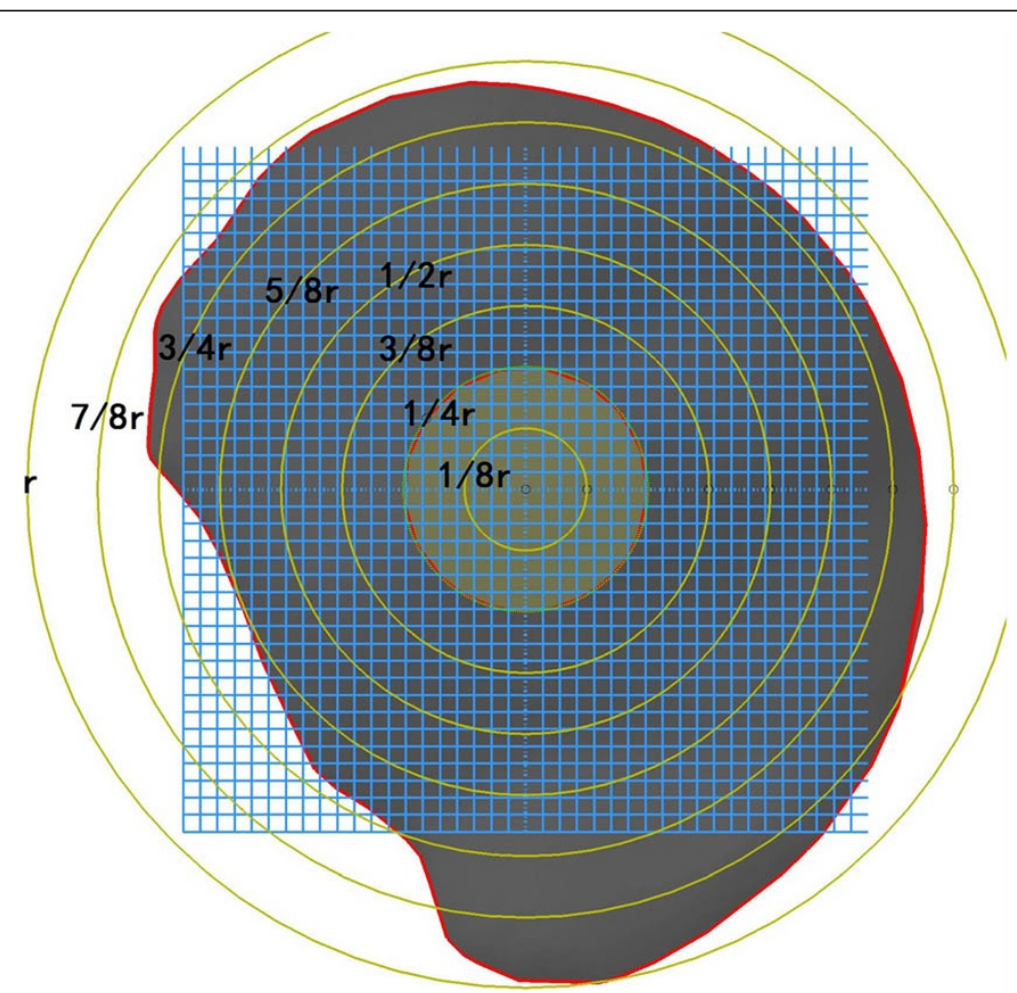

Fig. 3 Debridement size of necrotic lesion 


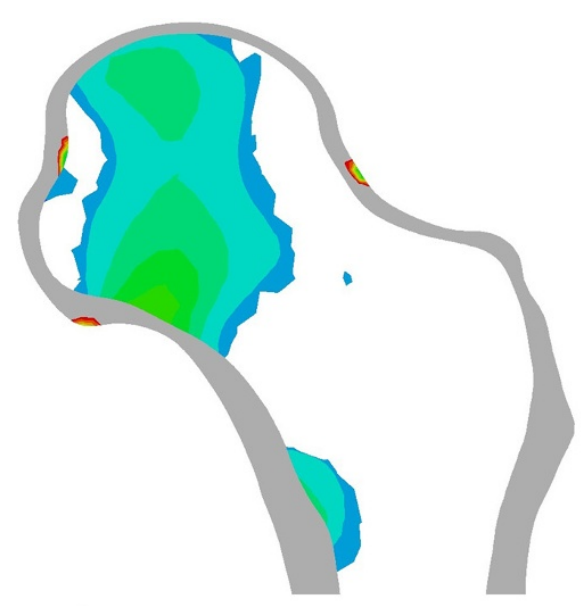

A Healthy simulation of P1

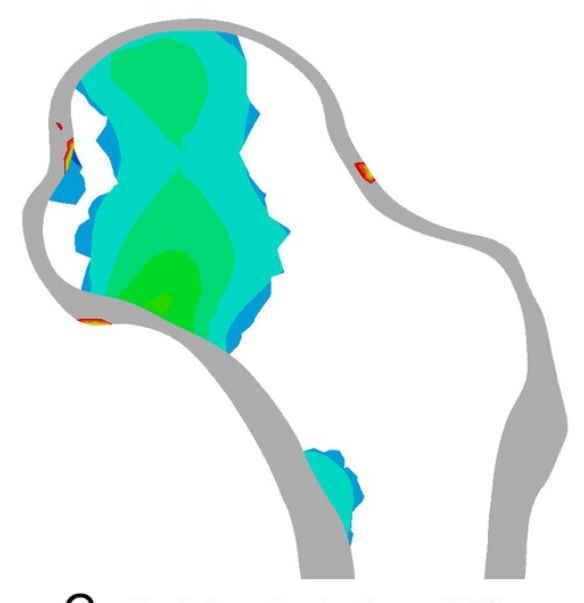

C Healthy simulation of P2

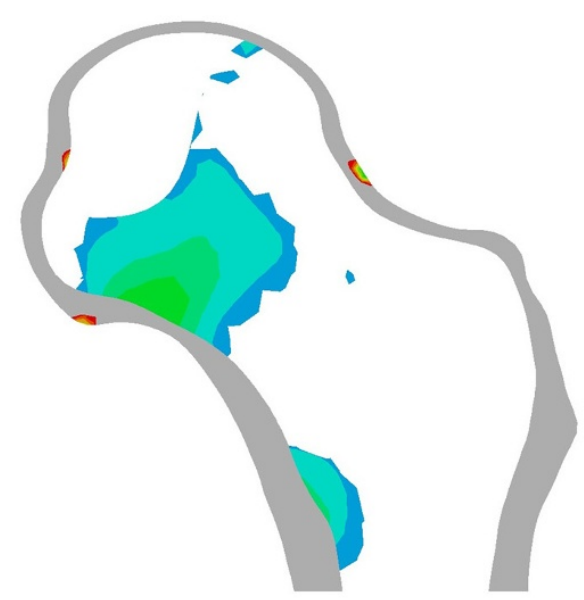

B Type $\mathrm{C1}$ of FHN

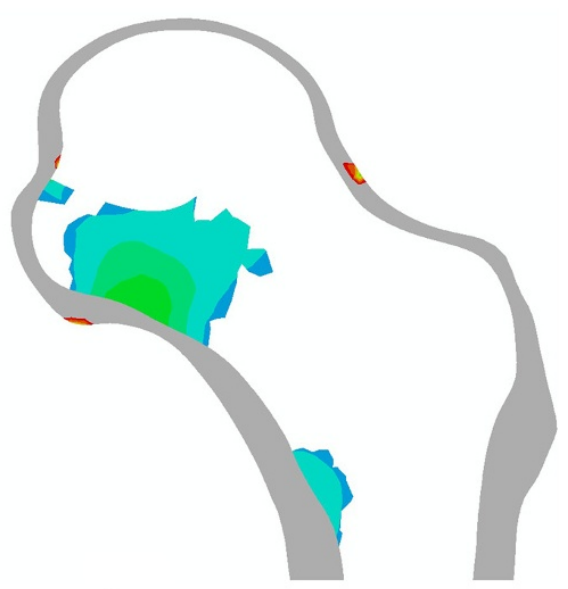

D Type C2 of FHN

Fig. 4 Principal stress distributions in the femoral head

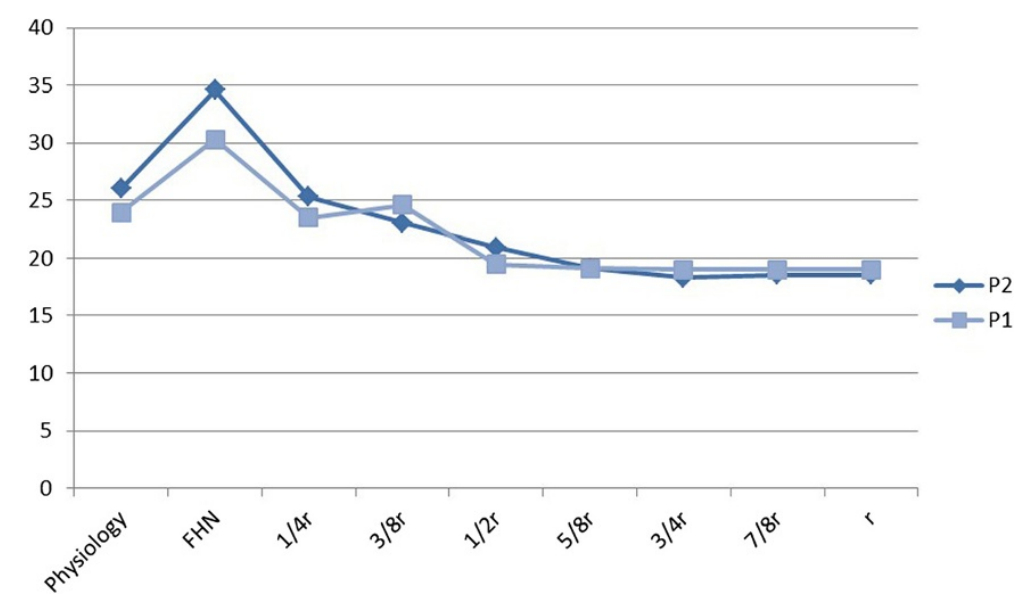

Fig. 5 Relationship between anterolateral stress and debridement region 
were $23.95 \mathrm{MPa}$ in P1 and $25.99 \mathrm{MPa}$ in P2. The JIC C1 FHN stress of $30.31 \mathrm{MPa}$ increased by approximately $26.56 \%$, which is higher than that obtained in the healthy condition (P1). The JIC C2 FHN stress of $34.58 \mathrm{MPa}$ increased by approximately $33.05 \%$, which is higher than that obtained in the healthy condition (P2). There is an obvious stress concentration region in the anterolateral column of the necrotic femoral head. When the debridement radius was $1 / 4 \mathrm{r}$, the stress was 23.52 $\mathrm{MPa}$ in $\mathrm{P} 1$ and $25.31 \mathrm{MPa}$ in $\mathrm{P} 2$, which are approximately $22.4 \%$ and $26.81 \%$ lower than those obtained in the JIC C1 (P1) and the JIC C2 conditions (P2), respectively. The peak stresses of the two postoperative cases returned to the near-healthy levels. After the FAIBG procedure, the stress concentration regions disappeared. When the debridement radius was greater than $3 / 8 \mathrm{r}$, the peak stress does not significantly change with an increase in the debridement radius.

\section{Peak stress of the residual necrotic bone}

Figure 6 demonstrates that the debridement size affects the stress distribution in the residual necrotic bone. Seven different necrotic debridement sizes ranging from $1 / 4 \mathrm{r}$ to $\mathrm{r}$ were selected to study the effect of the debridement radius on the residual necrotic bone. The relationship between the debridement size and the stress of the residual necrotic bone is shown in Fig. 5. When the debridement radius was $1 / 4 \mathrm{r}$, the peak stress increased by $3762 \%$ and $1217 \%$ compared with the values obtained in the JIC C1 and JIC C2 conditions, respectively. When the debridement was at least $3 / 8 \mathrm{r}$, the peak stress in the residual lesion rapidly decreased and returned to the physiological level.

\section{Efficiency of debridement}

The average stresses were calculated from all of the elements on the necrotic region and anterolateral column. The FAIBG procedure effectively decreased the average stress of the anterolateral column. However, a low-stress region gradually increased in the anterolateral column with an increase in the debridement radius. The load

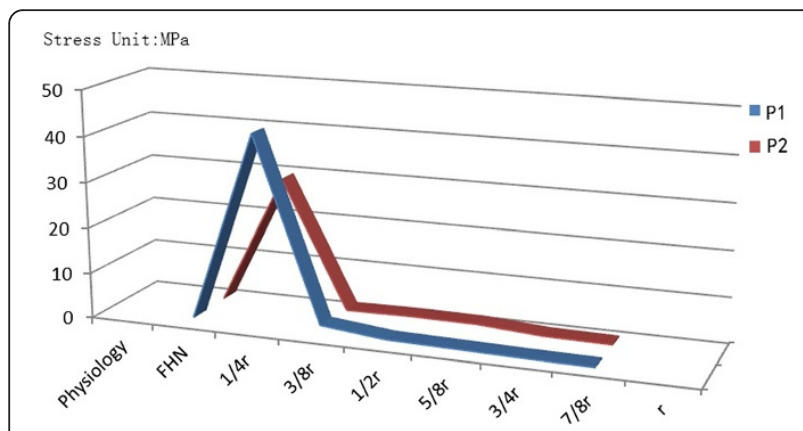

Fig. 6 Peak stress of the residual necrotic bone

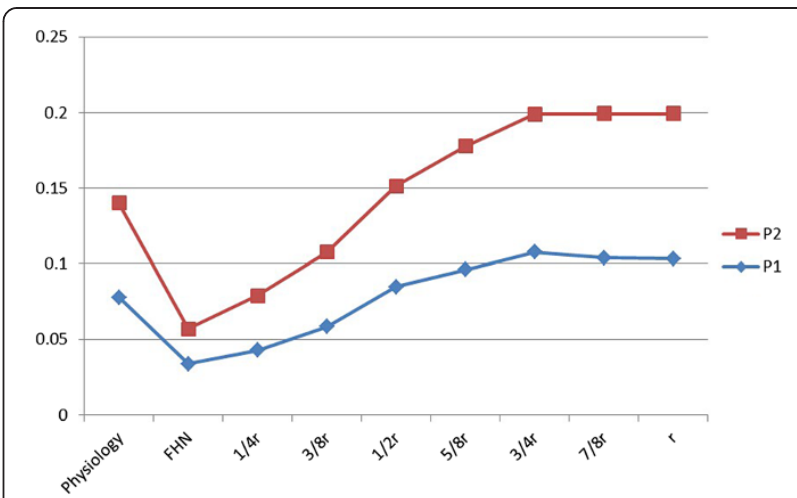

Fig. 7 Load share ratios based on different debridement regions

share ratio (LSR) is defined as the ratio of the average necrotic region stress to the average anterolateral column stress, which represents the bearing capacity of different material models in the femoral head. Fig. 7 displays the relationship between the necrotic region and anterolateral column based on different debridement regions. The bearing capacity of the necrotic region in the untreated condition is markedly decreased. The LSR increased with an increase in the debridement radius. In particular, when the debridement radius was larger than $1 / 2 r$, the LSR was larger than that obtained in the normal condition, which indicates that a proper debridement region may eliminate the stress concentration, but if the debridement region is too large, it may introduce the stress-shielding phenomenon.

\section{Model validation}

The principal compressive trabecula loads the principal compressive stress of the femoral head (Figs. 8c and d), which correlates well with the bone density distribution (Fig. 8b) [17]. The shape and location of the biomechanical transfer path for both load cases are consistent with the trabecular features in the cross-sections of the cadaver bone (Fig. 8a) [18, 19]. The trabeculae in the corresponding areas are clearly thinner. Simultaneously, the simulation results of our study (Figs. 4a and c) and the results of previous studies presented in the literature [13] have strongly similar stress patterns. Hence, we hypothesize that the finite element results can reflect the physical phenomenon of the hip and evaluate the results.

\section{Discussion}

Allograft bone is currently the most commonly used materials for the hip preserving procedure of FHN. Allograft fibula is used to provide structure and biomechanical support and impaction cancellous bone was used as biological repair material to promote osteogenesis during bone healing. The FAIBG procedure, as one of allograft bone 

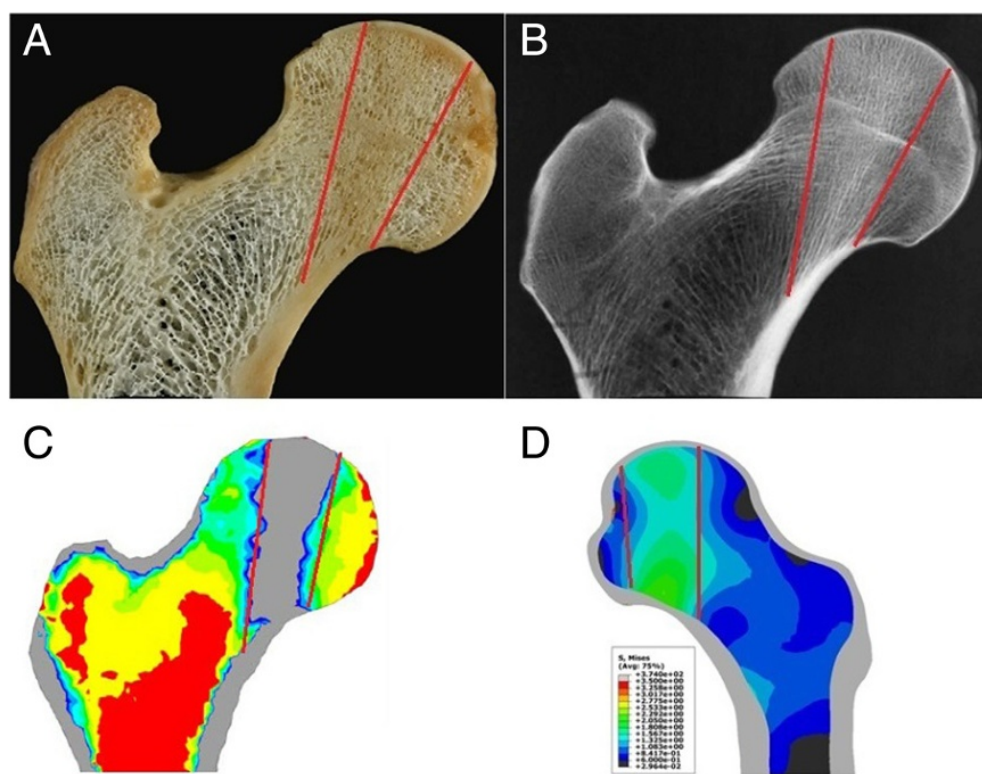

Fig. 8 Photograph (a), radiograph (b), the previous simulation results (c) and the computational results (d) of the human proximal femur investigated in this study

methods, represents a proven technique to maintain the shape of the femoral head and reduce the risk of FHC in its early stages. Rosenwasser [20] first described thorough debridement and bone grafting for the treatment of FHN in 1994. This technique is an effective method for young patients with early stage FHN, which delays the progression of osteoarthrosis and subsequent HR. Tao [21] reported an $80 \%$ clinical success rate with a mean follow-up time of 24 months among fifteen patients who had surgical therapy with thorough debridement with bone grafting. However, these procedures may cause serious artificial damage and complications because of capsulotomies or the destruction of the cortical bone of the femur neck fundus and require relatively high-cost and complicated technique. In 2008, Shi [22] reported 67 hips subjected to internal bracket implanting with partial debridement for FHN. These researchers showed a $64.2 \%(43 / 67)$ success rate with an average follow-up of 23 months. In 2013, Shi [23] treated 25 patients using an allograft fibula with partial debridement for FHN and reported satisfactory results in 18 of 25 (72\%) patients with a 24-month follow-up. These minimally invasive procedures could reduce the artificial damage and complications but result in a poorer clinical outcome because they cannot provide both repaired materials and biomechanical structural support during healing of the necrosis region. FAIBG with proper debridement is an effective head-preservation method, and we achieved an average clinical success rate of $90.3 \%$ with a mean follow-up time of 37.5 months [24]. All views are based on clinical observation experience and lack a biomechanical basis. Hence, both "thorough debridement" and "partial debridement" are not universally accepted because no compelling evidence indicates which method is better at reducing the collapse risk of the femoral head, which encourages us to apply our experiences to a computational biomechanical analysis of the extent of debridement to reduce the collapse risk of FHN.

In our study, we adopted a subject-specific computational approach to consider the changes in the stress distribution of the anterolateral cortical bone and residual necrotic bone. Fig. 4 shows that the stress transfer paths in both JIC C1 and C2 are completely broken off, which indicates that surgical intervention should be performed. The effect of the debridement size with FAIBG on the collapse risk is clearly demonstrated in Fig. 5. After FAIBG, the peak stress of the anterolateral cortical bone in all conditions could return to the physiological level, and in two cases, the decrement/increment in stress was almost less than $0.1 \%$ when the debridement radius increased. Hence, the collapse risk of the femoral head can be effectively reduced using an allo-fibula support to bear the load. Ueo [28] reported that the concentrated stress around the residual necrotic bone may induce development of the disease. When the debridement size is at least $3 / 8 \mathrm{r}$, the peak stress of the residual necrotic bone also returns to the pathological level, which denotes that the progression of necrosis will not deteriorate after surgical intervention. Fig. 7 shows that a proper debridement region may eliminate the stress concentration, but if the debridement region is too large and the bone grafting provides an oversized support intensity, 
the stress shielding phenomenon may be introduced. According to Wolff's law, the structure and function of bone are interdependent. Stress shielding may cause disused bone loss of the anterolateral cortical bone, which results in fracture and collapse. Our results provide specific biomechanical evidence to support the viewpoint that FAIBG with a debridement region of 3/8 - 1/2 appears to be a better choice for resisting the collapse of JIC C FHN.

Thorough debridement has been reported in previous studies [20, 21, 25-27]. However, this procedure is difficult and time-consuming and is associated with serious artificial damage. Simultaneously, thorough debridement and bone grafting may cause stress shielding, which results in fracture and collapse. FAIBG with partial debridement can eliminate stress concentration and stress shielding and ensure that the stress of the residual bone does not increase. This technique has a distinct biomechanical basis, saves time, requires relatively lower-cost and introduces a low risk of artificial damage. Hence, FAIBG without thorough debridement appears to be better than FAIBG with thorough debridement.

\section{Conclusions}

In this study, we propose using computational biomechanical technology to explore different mechanical performances of FAIBG with and without thorough debridement in order to provide a biomechanical basis for selecting the proper treatment in clinic settings. Eighteen computational models were constructed to simulate two subtypes of FHN with seven debridement radii during the FAIBG procedure. The simulation results provide specific biomechanical evidence to support the finding that FAIBG with a debridement region of $3 / 8-1 / 2$ appears to be a better choice for resisting the collapse of JIC C FHN. Furthermore, FAIBG without thorough debridement, which requires relatively simple technique and reduces artificial damage, appears to be a better method for resisting the collapse of JIC C1 and JIC C2 FHN. This manuscript also presents a preliminary approach to investigate the FAIBG procedure with thorough debridement, and a more detailed analysis will be reported in the near future.

\section{Competing interests}

The authors declared no potential conflicts of interest with respect to the research, authorship, and/or publication of this article.

\footnotetext{
Authors' contributions

ZGQ, HW, PZH and CXM have contributed to develop protocol of the study. $Z G Q, Z Y$ and ZLH have carried out data collection. ZGQ and ZY performed the experiments. ZGQ and ZLH have conducted data analysis. ZGQ and ZY wrote the paper. ZGQ, ZLH, XYJ, LSS and CLL translated the paper. ZGQ, HW, $\mathrm{PZH}$ and $\mathrm{CXM}$ have contributed to results interpretation and have read and approved the final manuscript.
}

Authors' Information

Guangquan Zhou, Ying Zhang, Linghong Zeng: co-first authors.

\section{Acknowledgements}

This study was supported by the National Science Foundation of China $(81173284,81473697)$ and the Natural Science Foundation of Guangdong Province (2014A030310214, S2013010011992). None of the authors of this paper have any financial and personal relationships with other people or organizations that could inappropriately influence the work.

\section{Author details}

'The First Affiliated Hospital, Guangzhou University of Chinese Medicine, Guangzhou, China. '2Laboratory of National Key Discipline Orthopaedics and Traumatology of Chinese Medicine, Guangzhou University of Chinese Medicine, Guangzhou, China. ${ }^{3}$ Department of Rheumatology, Guangdong Provincial Hospital of Chinese Medicine, China and Postdoctoral Mobile Research Station, Guangzhou university of Chinese Medicine, Guangzhou, China. ${ }^{4}$ Luoyang Orthopedic-Traumatological Hospital, Henan, China. ${ }^{5}$ Department of Rehabilitation Medicine, The 3rd People's Hospital, Huizhou, China, 516002. ' Department of Information Systems, City University of Hong Kong, Hong Kong, China.

Received: 14 November 2014 Accepted: 20 May 2015

Published online: 10 June 2015

\section{References}

1. Chan KL, Mok CC. Glucocorticoid-Induced avascular bone necrosis: Diagnosis and management. Open Orthop J. 2012;6:449-57.

2. Weinstein RS. Glucocorticoid-induced osteonecrosis. Endocrine. 2012;41(2):183-90.

3. Matuso K, Hirohata T, Sugioka Y, Lkeda M, Fukuda A. Influence of alcohol intake, cigarette smoking, and occupational status on idiopathic osteonecrosis of the femoral head. Clin Orthopm. 1988;234:115-23.

4. Hirota Y, Hirohata T, Fukuda K, Mori M, Yanagawa H, Ohno Y, et al. Association of alcohol intake, cigarette smoking and occupational status with the risk of idiopathic osteonecrosis of the femoral head. Am J Epidemiol. 1993;137(5):530-8.

5. Wang Y, Li Y, Mao K, Li J, Cui Q, Wang GJ. Alcohol-induced adipogenesis in bone and marrow: a possible mechanism for osteonecrosis. Clin Orthop Relat Res. 2003:410:213-24.

6. Shigemura T, Nakamura J, Kishida S, Harada Y, Takeshita M, Takazawa M, et al. The incidence of alcohol-associated osteonecrosis of the knee is lower than the incidence of steroid-associated osteonecrosis of the knee: an MRI study. Rheumatology. 2012;51(4):701-6.

7. Martin RW, Manfred H, Janos V, Ncholas DS. Influence of polymer surface chemistry on frictional properties under protein-lubrication conditions: implications for hip-implant design. Tribol Lett. 2001;10(1-2):111-6.

8. Brannon JK. Influence of acetabular coverage on hip survival after free vascularized fibular grafting for femoral head osteonecrosis. J Bone Joint Surg Am. 2007;89:448-9.

9. Katz MA, Urbaniak JR. Free vascularized fibular grafting of the femoral head for the treatment of osteonecrosis. Tech Orthop. 2001;16:44-60.

10. Malizos KN, Soucacos PN, Beris AE. Osteonecrosis of the femoral head. Hip salvaging with implantation of a vascularized fibular graft. CLin Orthop Relat Res. 1995;314:67-75.

11. Urbaniak JR, Coogan PG, Gunneson EB, Nunley JA. Treatment of osteonecrosis of the femoral head with free vascularized fibular grafting. A long-term follow-up study of one hundred and three hips. J Bone Joint Surg Am. 1995;77:681-94.

12. Sugano N, Atsumi T, Ohzono K, Kubo T, Hotokebuchi T, Takaoka K. The 2001 revised criteria for diagnosis, classification, and staging of idiopathic osteonecrosis of the femoral head. J Orthop Sci. 2002;7:601-5.

13. Sverdlova NS, Witzel U. Principles of determination and verification of muscle forces in the human musculoskeletal system: Muscle force to minimise bending stress. J Biomech. 2010;43:387-96.

14. Brown TD, Hild GL. Pre-collapse stress redistributions in femoral head osteonecrosis-a three-dimensional finite element analysis. J Biomech Eng. 1983:105:171-6.

15. Brown TD, Way ME, Ferguson Jr AB. Mechanical characteristics of bone in femoral capital aseptic necrosis. Clin Orthop Relat Res. 1981;156:240-7.

16. Grecu D, Pucalev I, Negru M, Tarnita DN, Ionovici N, Dita R. Numerical simulations of the 3D virtual model of the human hip joint, using finite element method. Rom J Morphol Embryol. 2010;51:151-5. 
17. Jang IG, Kim IY. Computational study of Wolff's law with trabecular architecture in the human proximal femur using topology optimization. J Biomech. 2008;41:2353-1361.

18. Christopher B, Kim IY. Three-dimensional micro-level computational study of Wolff's law via trabecular bone remodeling in the human proximal femur using design space topology optimization. J Biomech. 2011;44:935-42.

19. Jang IG, Kim IY. Computation simulation of trabecular adaptation progress in human proximal femur during growth. J Biomech. 2009:42:573-80.

20. Rosenwasser MP, Garino JP, Kiernan HA, Michelsen CB. Long-term follow up of thorough debridement and cancellous bone grafting for osteonecrosis of the femoral head. Clin Orthop. 1994;306:17-27.

21. Tao W, Wei W, Zong SY. Treatment of osteonecrosis of the femoral head with thorough debridement, bone grafting and bone-marrow mononuclear cells implantation. Eur J Orthop Surg Traumatol. 2014;24:197-202.

22. Shi FL, Lu FX, Li XH, Huang Y, Zhang MC. Clinical observation on internal bracket implanting for treatment of adult necrosis of femoral head and finite element analysis. Chinese journal of bone and joint injury. 2008;23(3):186-8

23. Shi FL, Chen J, Li XH, Lu FX. Fan-shaped decompression and allograft fibula supporting internal fixation for treatment of early femoral head necrosis in adults. Chinese journal of tissue engineering research. 2013;17(44):7758-63.

24. He W, Yong L, Zhang QW, Wang HB, Fang B, Pang ZH, et al. Primary outcome of impacting bone graft and fibular autograft or allograft in treating osteonecrosis of femoral head. Chinese Journal of Reparative and Reconstructive Surgery. 2009;23(5):530-3.

25. Ueo T, Tsutsumi S, Yamamuro T, Okumura H, Shimizu A, Nakamura T. Biomechanical aspects of the development of aseptic necrosis of the femoral head. Arch Orthop Trauma Surg. 1985;104:145-9.

26. Meyers MH, Jones RE, Bucholz RW, Robert W, Wenger DR. Fresh autogenous grafts and ostaochondral altografts for the treatment of segmental collapse in osteonecrosis of the hip. Clin Orthop. 1983;174:10-2.

27. Ko JY, Meyers MH, Wanger DR. "Trapdoors" Procedure for osteonecrosis with segmental collapse of the femoral head in teenagers. J Padiatt Orthop. 1995;15:7-15.

28. Meyers MH, Convery FR. Grafting procedure in osteonecrosis of the hip. Sem in Arthroplasty. 1991;2:189-97.

\section{Submit your next manuscript to BioMed Central and take full advantage of:}

- Convenient online submission

- Thorough peer review

- No space constraints or color figure charges

- Immediate publication on acceptance

- Inclusion in PubMed, CAS, Scopus and Google Scholar

- Research which is freely available for redistribution 